\section{Precursors of 'bad' osteoclasts identified in arthritis}

Osteoclasts are important for both homeostatic bone remodelling in health and pathological bone erosion in rheumatoid arthritis (RA), but whether different types of osteoclasts mediate these two processes is unclear. New findings suggest that osteoclasts in these different tissue settings arise from different precursor cells and hence follow distinct developmental trajectories.

To identify osteoclast precursor cells in the synovium, researchers in a new study developed a protocol that enabled them to isolate synovium from 'bare areas' of the joint (areas where the synovium comes into contact with bone). They detected a subpopulation of macrophages (termed arthritis-associated osteoclastogenic macrophages (AtoMs)) in the inflamed synovium of mice with collagen-induced arthritis (CIA). This population contained osteoclast precursors that were phenotypically distinct from osteoclast precursors involved in homeostatic bone remodelling in the bone marrow.

Transcriptional profiling implicated the transcription factor FOXM1 as an important regulator of AtoMs. Indeed, treatment with thiostrepton (an inhibitor of FOXM1 activity) inhibited osteoclastogenesis of AtoMs in vitro. In mice with CIA, thiostrepton treatment reduced the arthritis scores of the mice and inhibited the expression of pro-inflammatory cytokines in the synovium. However, in healthy mice, thiostrepton had no effect on homeostatic bone remodelling.

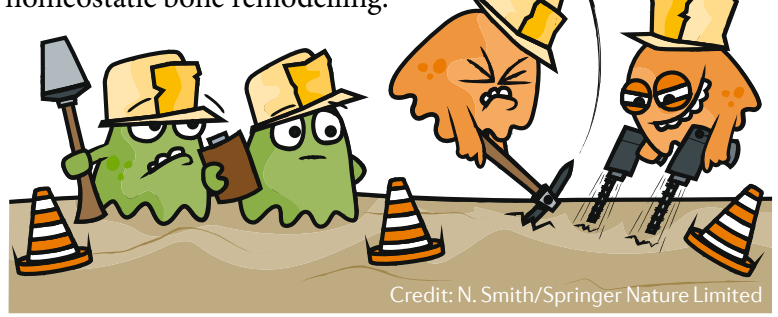

In mice with collagen antibodyinduced arthritis, tamoxifen-mediated deletion of Foxm1 alleviated bone erosion and partially reduced the arthritis scores of the mice. Adoptive transfer of Foxm1-expressing monocytes partially reversed these effects.

Notably, the researchers identified a population of macrophages in the synovium of patients with RA that corresponded to mouse AToMs. The cells expressed FOXM1 and had a high osteoclastogenic potential that was inhibited with thiostrepton in vitro.

The researchers plan to develop a novel therapy that specifically targets these 'bad' osteoclast precursors.

"Because current regimens for treating bone diseases block both 'good' and 'bad' osteoclasts, a new line of therapy targeting only 'bad' ones would be meritorious for future patient care," explains corresponding author Masaru Ishii.

\section{Jessica McHugh}

ORIGINAL ARTICLE Hasegawa, T et al.

Identification of a novel arthritis-associated osteoclast precursor macrophage regulated by FoxM1. Nat. Immunol. 20, 1631-1643 (2019)

\title{
Pathogenicity of neutrophils linked to maturation stage in SLE
}

Dysregulated neutrophils have a pathogenic role in systemic lupus erythematosus (SLE), in particular through the release of neutrophil extracellular traps (NETs) and the subsequent production of type I interferon. However, exactly how neutrophils become dysregulated in SLE is an active area of research.

"Our group had previously identified a pro-inflammatory neutrophil subset known as low-density granulocytes (LDGs). We found that these cells could damage blood vessels and promote

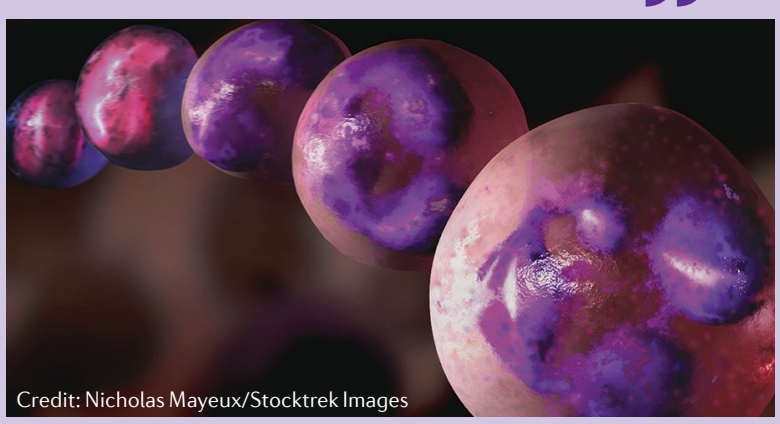

intermediatemature LDGs in the blood of patients with SLE correlated with more severe organ damage

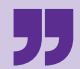

immune dysregulation in SLE," explains Mariana Kaplan, corresponding author of a new study on LDGs. pathogenic features of LDGs and was responsible for the type I interferon gene signature in SLE," says Kaplan.

Interestingly, high numbers of intermediate-mature LDGs in the blood of patients with SLE correlated with more severe organ damage, reduced kidney function and an increased coronary plaque burden. The results of further in vivo and in vitro studies also suggested a role for these cells in vascular damage.

"Future studies will focus on identifying potential therapies that can specifically target the intermediate-mature LDG subset in the treatment and/or prevention of lupus vasculopathy and premature cardiovascular disease in SLE," states Kaplan.

Joanna Clarke

ORIGINAL ARTICLE Mistry, P. et al. Transcriptomic, epigenetic, and functional analyses implicate neutrophil diversity in the pathogenesis of systemic lupus erythematosus. Proc. Natl Acad. Sci. USA 116, 25222-25228 (2019) the intermediate-mature subpopulation of LDGs displayed the bulk of the subsets by examining their ability to perform typical neutrophil tasks such as degranulation, NET production and phagocytosis. "Overall, we found that and that could be distinguished CD10-immature LDGs and CD10 intermediate-mature LDGs. The researchers went on to functionally characterize these the heterogeneity of LDGs and whether subsets existed with distinct and epigenomic approaches to with SLE. Notably, they identified two subsets that seemed to represent 